\title{
Sarcoidosis with infiltration of the external ocular
} muscles

\author{
K STANNARD AND D J SPALTON \\ From the Medical Eye Unit, St Thomas's Hospital, London SE1
}

SUMmaRY This is a clinicopathological report of a patient with systemic sarcoidosis who had infiltration of the external ocular muscles, Tenon's capsule, and lacrimal glands.

Sarcoidosis is recognised as a multisystem disease of unknown aetiology in which non-caseating granulomas are found in the individual organs and the clinical presentation depends on site, quantity, and duration of the granulomatous infiltration within the affected organ. About $25 \%$ of patients with sarcoidosis have ophthalmic involvement manifested either by infiltration of the ocular adnexa, intraocular inflammation, or infiltration of the retrobulbar visual pathways. Orbital disease with sarcoidosis is rare. We have seen occasional patients presenting with orbital apex or cavernous sinus syndromes, but a discrete orbital granuloma or myositis appears to be exceptional.

In a review of the ophthalmic manifestations of sarcoidosis Obenauf et al. recorded orbital disease in only two out of 532 patients, but did not describe these cases further.' Trokel and Hilal reviewed 70 cases of orbital myopathy diagnosed from CT scan appearances, but did not have any cases of sarcoidosis in their series. ${ }^{2}$ In contrast, skeletal muscle myopathy with sarcoidosis is well recognised and presents in overlapping patterns of palpable muscle nodules, acute polymyositis, or a chronic myopathy. ${ }^{35}$ The majority of these patients are asymptomatic, and it is possible that the incidence of skeletal muscle disease is underestimated, as biopsies are rarely performed on asymptomatic patients. Some authors have estimated that $50 \%$ or more of patients with systemic sarcoidosis have histologically identifiable granulomata between normal muscle fibres. ${ }^{56}$

We present a case history with CT scans, operative photography, and confirmatory histology of a patient with marked sarcoid infiltration of an external ocular muscle.

Correspondence to D J Spalton, FRCS, St Thomas's Hospital, London SE1 7EH. No reprints available.

\section{Case report}

In May 1980 a 39-year-old West Indian man presented to the Ophthalmic Department of St Thomas's Hospital with a one-month history of a lump in the outer aspect of the upper eyelid, which had been slowly enlarging and had recently been tender to touch. He had no visual disturbance and, other than occasional aching in his chest, no other systemic symptoms.

On examination he was a fit man with normal cardiovascular, respiratory, and abdominal systems. There were a few shotty, slightly tender lymph nodes in his right groin. His visual acuities were $6 / 5$ in either eye. There was a soft, mobile mass in the outer aspect of the left upper eyelid (Fig. 1), which had the clinical features of a lacrimal gland swelling. There was no displacement of the globe, and eye movements were full in all directions. Examination of the anterior segments and fundus was normal.

Although an $x$-ray of the skull and orbits showed no abnormality, a chest $x$-ray demonstrated rightsided hilar lymphadenopathy, which was confirmed by hilar tomography and was compatible with a diagnosis of sarcoidosis. Routine haematological and biochemical analyses were all normal, apart from showing sickle cell trait with a haemoglobin phenotype of $\mathrm{Hb}$ SA. Mantoux skin testing was negative to a dilution of $1: 100$, but a Kveim test was strongly positive, and a diagnosis of presumed sarcoidosis with lacrimal gland involvement was made.

In June 1980, because of increasing tenderness in the groin, he underwent excision biopsy of a lymph node. This showed 'foci of epithelioid cells and Langhan's giant cells, with no evidence of acid fast bacilli, consistent with sarcoidosis.' In the absence of pulmonary infiltration the patient was followed up without treatment. 


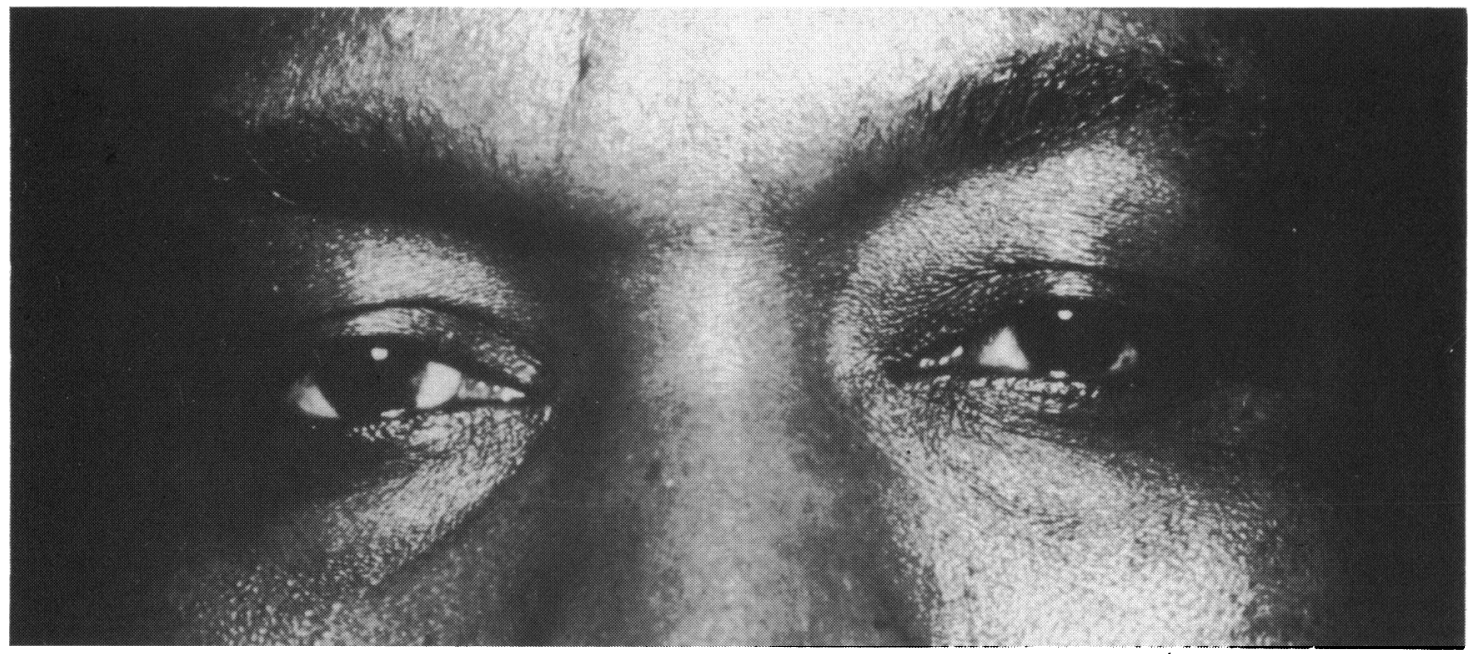

Fig. 1 Face photograph of the patient showing enlargement of the left lacrimal gland.

Eight months later the patient complained of redness of the left eye. On examination there was an area of fleshy episcleritis over the distal portion of the medial rectus muscle, with prominent vessels over a mass which seemed to be lying on or in the muscle belly (Fig. 2). Ocular movements were full and there was no diplopia. The lacrimal gland swelling was unchanged. Orbital CT scans (Figs. 3, 4) showed thickening of the left medial rectus muscle and lacrimal gland on axial CT scans (Fig. 3) and marked infiltration of the insertions of the other recti and Tenon's capsule on coronal scans. Because of these

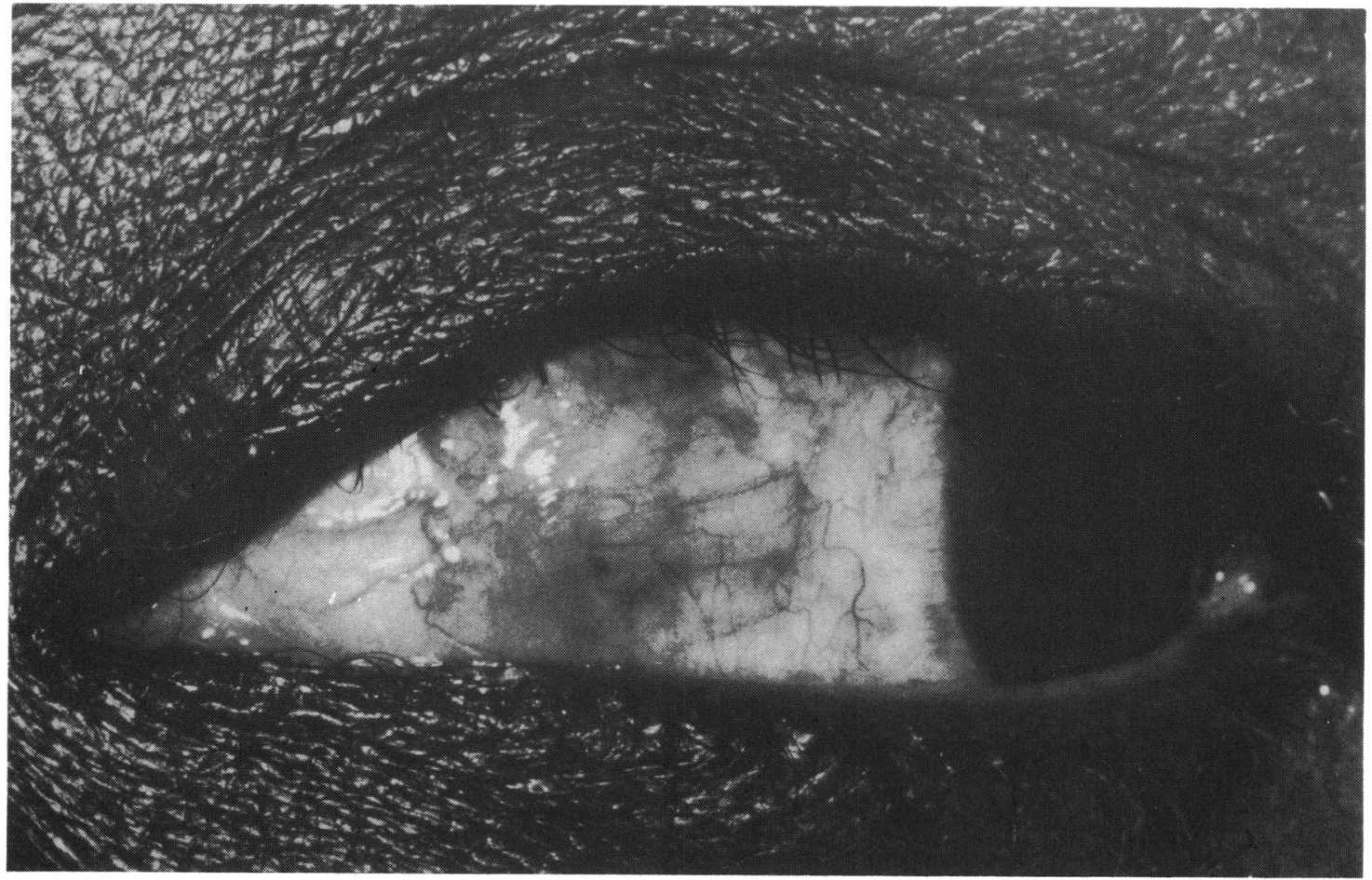

Fig. 2 Eight months later: close-up photograph of the left eye shows an inflamed subconjunctival mass in the region of the medial rectus tendon. 


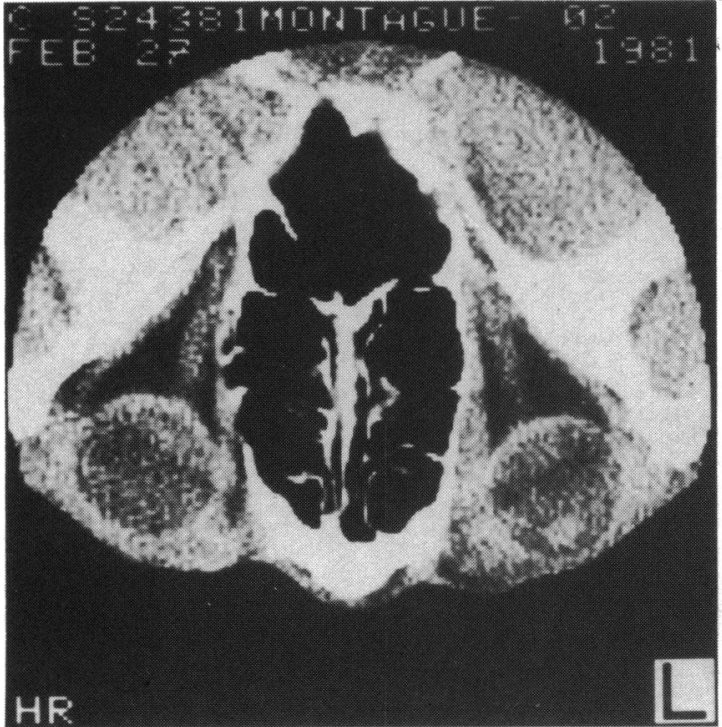

Fig. 3 Enhanced axial CT scan shows thickening of left medial rectus muscle and swelling at its insertion with enhancement of the Tenon's capsule in each eye and prominence of the lacrimal gland.

unusual features the patient underwent in March 1981 a transcutaneous biopsy of the left lacrimal gland swelling and exploration of the medial rectus muscle. The conjunctiva was reflected to reveal a grossly thickened muscle belly (Fig. 5) infiltrated by a firm pearly white mass, from which a specimen was

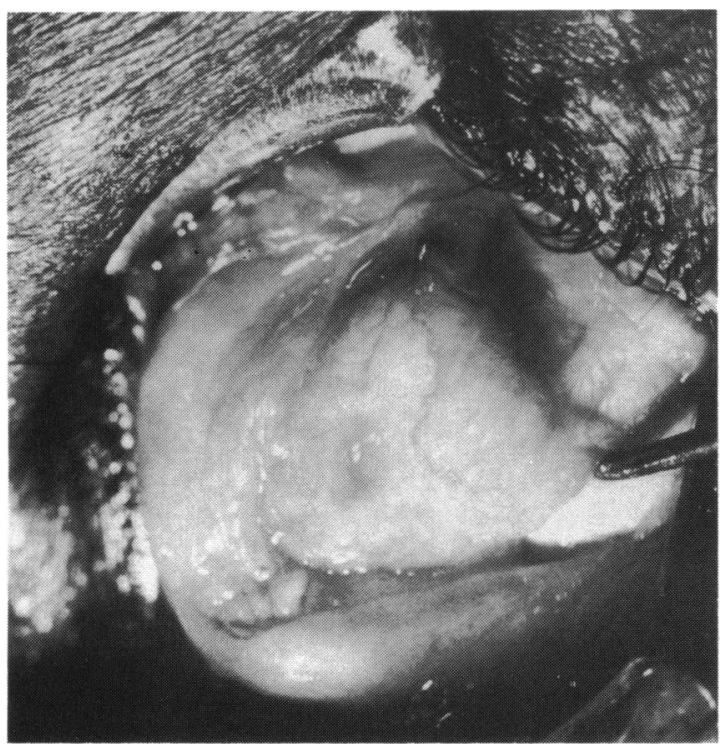

Fig. 5 Surgical photograph showing left medial rectus tendon lying over a squint hook. The tendon is thickened and infiltrated with pearly white tissue.

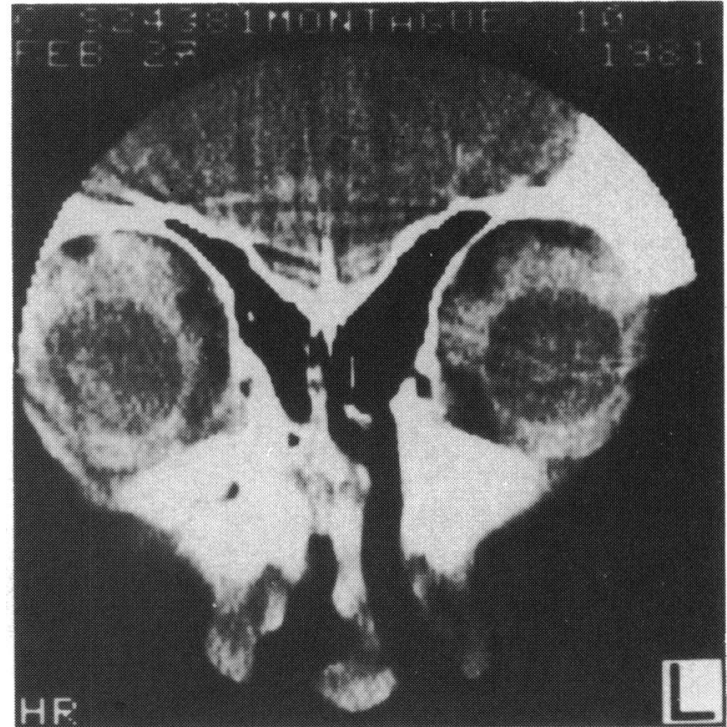

Fig. 4 Enhanced coronal CT scan shows an enhancing circumferential infiltration of Tenon's capsule surrounding the globe with thickening of the recti tendons.

taken for biopsy. Histologically the lacrimal gland showed multiple, non-caseating granulomata with epithelioid cells and Langhan's giant cells (Fig. 6). There was no sign of acid fast bacilli. Similarly, the muscle biopsy showed typical sarcoid granulomata, surrounded by degenerate muscle fibres (Fig. 7). The patient made an uneventful recovery.

On the biopsy results and clinical findings a diagnosis of multisystem sarcoidosis was made. He was successfully treated with a course of systemic steroids, which was gradually tapered off over a few months. He has since been lost to follow-up.

\section{Discussion}

The diagnosis of sarcoidosis in this patient is substantiated by the demonstration of pathological changes in the lymph nodes, lacrimal gland, and ocular muscle, and appropriate radiological signs on the chest $x$-ray, with a positive Kveim test. Uveitis is the most common ocular manifestation of sarcoidosis and is usually seen as an anterior uveitis. But posterior uveitis is common; it may occur in the absence of anterior uveitis and is the most frequent cause of visual morbidity. ${ }^{7}$ Involvement of the ocular adnexa from sarcoidosis is usually restricted to infiltration of the skin, conjunctivae, or lacrimal glands with granulomatous tissue. These sites are well recognised and provide good material for biopsy and histological confirmation of the diagnosis; 'blind' biopsy of apparently normal conjunctiva can be a 


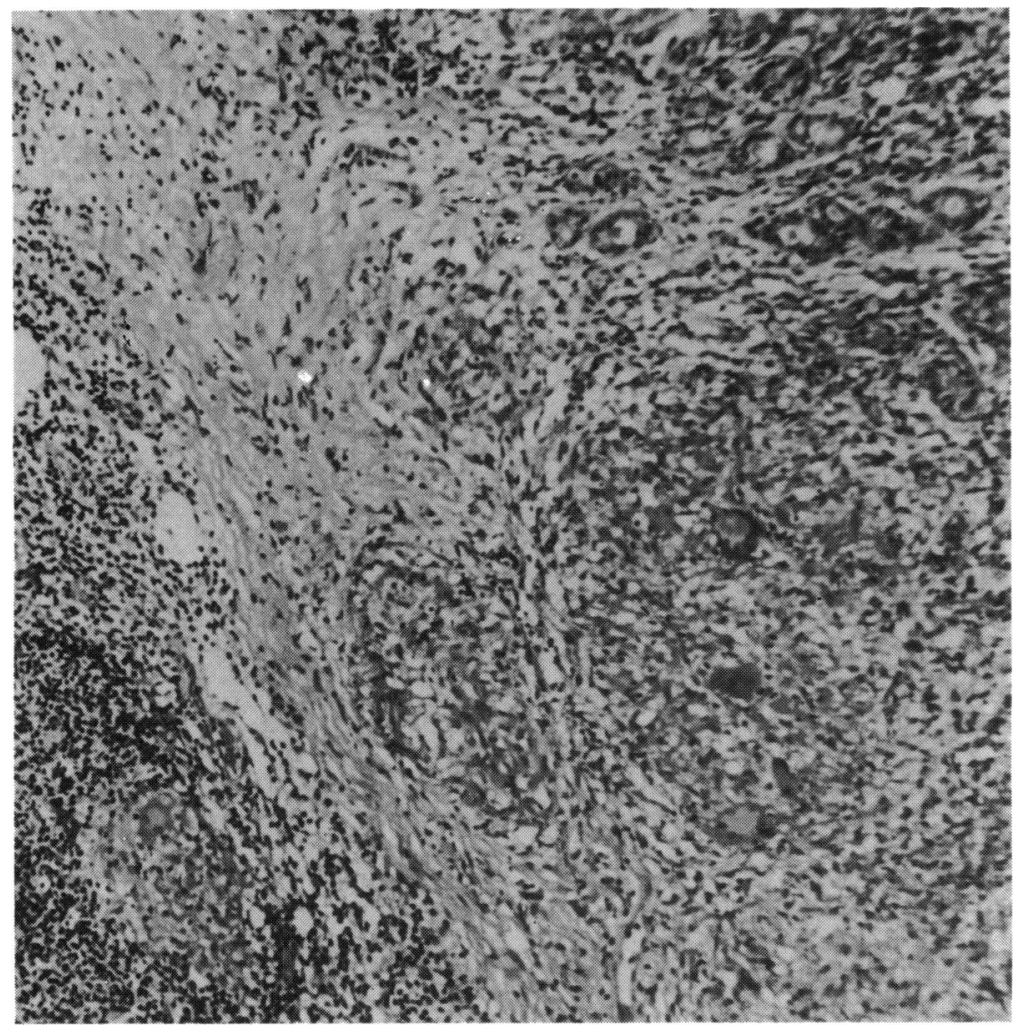

Fig. 6 Left lacrimal gland biopsy showing a typical sarcoidal granuloma with giant cells. (Haematoxylin and eosin.)

helpful diagnostic procedure. ${ }^{~}$ A lacrimal gland is involved in about $75 \%$ of patients with active acute sarcoidosis. If required, lacrimal gland biopsy should be performed transcutaneously, because a transcon-

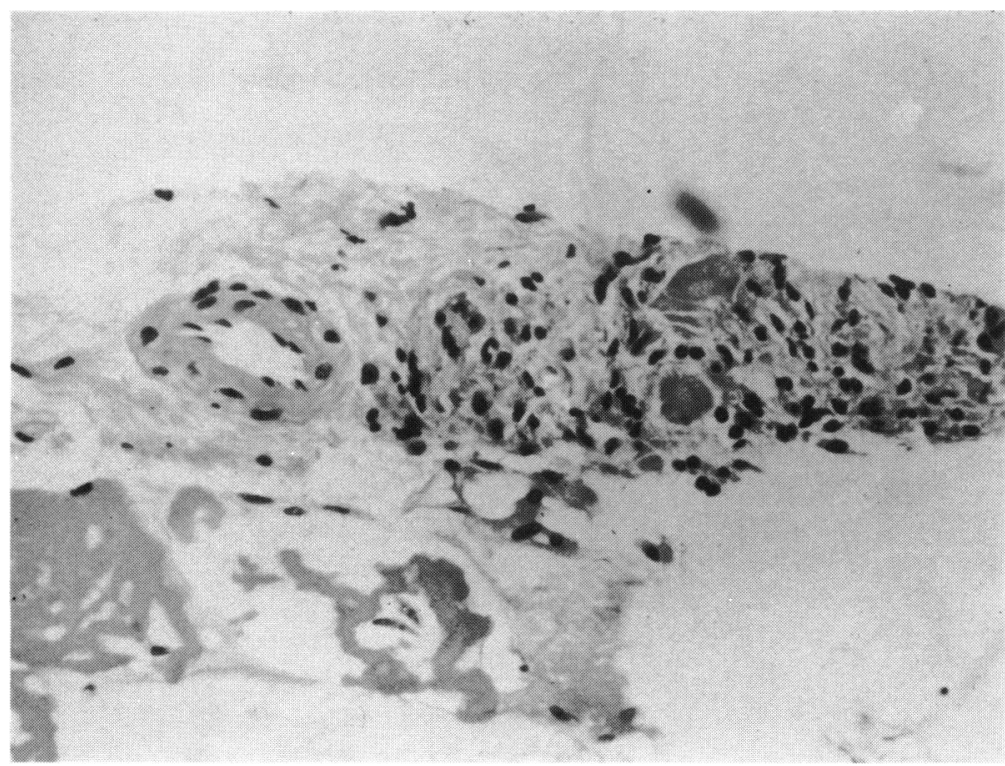

junctival approach may damage the ductules of the gland and risk producing a dry eye in a disease where this is already a possibility. In contrast to these manifestations sarcoidosis of the retrobulbar space is
Fig. 7 Biopsy of left medial rectus tendon shows chronic granulomatous infiltration with degenerate muscle fibres. (Haematoxylin and eosin.) 
uncommon, there being only one previous report published prior to the advent of CT scanning." We see an occasional superior orbital fissure or cavernous sinus syndrome from sarcoidosis in the Medical Eye Unit, but a myositis or discrete retrobulbar mass is most unusual.

This patient showed dramatic signs of involvement in the recti tendons and Tenon's capsule on his CT scans (Figs. 3, 4).

The differential diagnosis of thickening of the external ocular muscles lies between such conditions as endocrine exophthalmos, vascular congestion associated with arteriovenous fistulas, infiltration by tumours (e.g., meningioma), inflammatory myositis, and pseudotumour. The CT scan appearances are crucial in this differential diagnosis, and most of the conditions are readily identifiable. The differentiation of an inflammatory myositis from endocrine exophthalmos can be difficult, but is aided by demonstration of involvement of the muscle tendon by an inflammatory myositis in contrast to endocrine exophthalmos, where the hypertrophy is restricted to the muscle belly itself. Our patient provides an excellent demonstration of this sign. The CT scan shows gross changes in the muscle tendons and Tenon's capsule (Fig. 4), and the muscle tendon was found to be infiltrated at surgery (Fig. 5).

We have found only one previous report of orbital myositis from sarcoidosis, where this was reported as a coincidental finding in an eye enucleated for panuveitis. ${ }^{10}$ This seems at odds with the wealth of papers reporting skeletal muscle involvement, but might be explained by the fact that sarcoidal infiltration of skeletal muscle is often asymptomatic, and perhaps external ocular muscle involvement may be commoner than is realised. Fewer than $1 \%$ of patients with sarcoidosis have symptoms of myalgia, weakness, tenderness, or wastage, and symptomatic patients tend to have chronic disease. Indeed, if patients do complain of ocular pain and myalgia against a background of active uveitis, it is likely that the true cause of discomfort might not be recognised for what it is.

We thank Mr M D Sanders, who cared for the patient and agreed to allow us to publish the findings.

References

1 Obenauf CD, Shaw HE, Sydnar CF, Klintworth GK. Sarcoidosis and its ophthalmic manifestations. Am J Ophthalmol 1978; 86: 648-55.

2 Trokel SL, Hilal SK. Recognition and differential diagnosis of enlarged extraocular muscles in computed tomography. Am J Ophthalmol 1979; 87: 503-12.

3 Currie S. In: Walton SJ, ed. New York: Churchill Livingstone, 1981: 525-68. Inflammation myopathies. 4th ed.

4 Crompton MR, MacDermot V. Sarcoidosis associated with progressive muscular wasting and weakness. Brain 1961; 84: 62-74.

5 Douglas AC, MacLeod JG, Matthews JD. Symptomatic sarcoidosis of skeletal muscle. J Neurol Neurosurg Psychiatry 1973; 36: 1034-40.

6 Kagen LJ. Less common causes of myositis. Clin Rheum Dis 1984; 10: 175-87.

7 Spalton DJ, Sanders MD. Fundus changes in histologically confirmed sarcoidosis. Br J Ophthalmol 1981; 65: 348-58.

8 Nichols CW, Eagle RC, Yanoff M, Manocal NG. Conjunctival biopsy as an aid in the evaluation of the patient with suspected sarcoidosis. Ophthalmology (Rochester) 1980; 87: 287-91.

9 Melmon KL, Goldberg J. Sarcoidosis with bilateral exophthalmos as the presenting symptom. Am J Med 1962; 33: $158-60$.

10 Frank KW, Weiss $\mathrm{H}$. Unusual clinical and histopathological findings in ocular sarcoidosis. Br J Ophthalmol 1983; 67: 8-16. 